\title{
Changes to the Vibration Response of a Model Power Transformer with Faults
}

\author{
Yuxing Wang \\ College of Biomedical Engineering and Instrument Science, Zhejiang University, 38 Zheda Road, Hangzhou, Zhe- \\ jiang, China, 310027.
}

\begin{abstract}
Jie Pan
School of Mechanical and Chemical Engineering, the University of Western Australia, 35 Stirling HWY, Crawley, Western Australia, Australia, 6009.
\end{abstract}

(Received 13 October 2015; accepted 26 August 2016)

Current vibration-based techniques for transformer condition monitoring mostly rely on the vibration response caused by operating excitations, which consist of electrical excitations from the core and winding. Therefore, it is worthwhile to study the electrically-excited frequency response function (FRF), as it carries information of transformer mechanical and electromagnetic properties. This paper includes a sensitivity analysis of the mechanically and electrically excited FRFs of a model transformer to the reasons behind its failures. A model power transformer is used as an example to demonstrate the variation of its vibration response to a couple of causes of transformer faults, such as looseness of clamping forces in winding and core. Experimental evidence is presented to show the quantitative description of the causes of artificial faults and to extract features of variations of FRFs that might be useful to the vibration-based detection of the causes of transformer faults in general.

\section{INTRODUCTION}

In the power industry, monitoring health conditions and detecting the causes of power transformer failures are often done using one of three methods: dissolved gas analysis (DGA), frequency response analysis (FRA), and vibration-based methods. ${ }^{1-5}$ These methods focus on measuring the indicators of transformer faults and correlating the trends of changes in these said indicators with respect to the causes of transformer failures.

As an online and nonintrusive method, the vibration-based condition monitoring method has attracted considerable attention for transformer health monitoring in the past few decades. Previous work has demonstrated that this method provides an option for assessing the mechanical integrity of a transformer. $^{6-12}$ Unlike the DGA and FRA methods, it relies on changes in the vibration response of the transformer under both steady-state and transient processes. For an example, Berler found that looseness in the winding clamping force might cause variations at twice the operating frequency and its harmonics. ${ }^{6}$ The transient vibration evoked by transformer energize/de-energize operations was also employed to detect abnormalities in transformer winding. ${ }^{7}$ To further develop the vibration-based condition monitoring method, efforts were also made in the area of signal processing to extract the vibration features of a damaged transformer by advanced signal processing methods, including the wavelet transform, the Hilbert Huang transform, and their combinations. ${ }^{10}$

Although the feasibility of using the vibration method for transformer condition monitoring was verified in these case studies, there is still a gap in understanding the physical correlation between the changes in the vibration response and changes in the transformer's mechanical properties associated with the causes of failures. A better understanding of vibration changes caused by transformer mechanical faults is beneficial to fault allocation, even to the development of novel monitoring strategies, which is the research motivation of this work.

The vibration response of a power transformer is a measure of the transformer vibration (as outputs) with respect to the transformer's electrical inputs. Since the transformer online monitoring techniques mostly rely on the vibration response caused by operating excitations that consist of electrical excitations from core and winding, it is more straightforward to study the electrically excited FRF and its variations as opposed to the mechanical excitation. The previous work by Wang and Pan examined the vibration FRFs of a model power transformer to the electrical excitation. ${ }^{13}$ However, their changes to different failure causes and corresponding change sensitivities have not been inferred.

Because the vibration of the winding and core are nonlinear functions of the electrical inputs, the traditional concept of the frequency response function (FRF) for linear systems does not apply. In a previous work, it was found that the steadystate response was characterized by the frequency components at twice the excitation frequency and its harmonics. ${ }^{5}$ Therefore, the nonlinear vibration response of the transformer with respect to a sinusoidal input can still be specifically defined in the frequency domain. For example, if the secondary winding is in an open circuit condition, then the vibration response function is defined as:

$$
H\left(x_{i} \mid \omega\right)=\sum_{k=1}^{\infty} H_{2 k}\left(x_{i} \mid 2 k \omega_{o}\right) ;
$$

where $x_{i}$ was the measurement location of the vibration re- 
sponse, $\omega_{0}$ was the excitation frequency, and the frequency response component $H_{2 k}\left(x_{i} \mid 2 k \omega_{o}\right)=\frac{v\left(x_{i} \mid 2 k \omega_{o}\right)}{U\left(\omega_{o}\right)}$ was defined by the ratio of the vibration component $v\left(x_{i} \mid 2 k \omega_{o}\right)$ at $2 k \omega_{o}$ and the primary voltage amplitude at $\omega_{o}$. This definition of the input and output relationship of a transformer was practically significant as almost all in-service power transformers were excited by a sinusoidal voltage. For many practical applications, a large percentage of the vibration energy was contained at $\omega=2 \omega_{o}$. Therefore, the first term of the response function $H\left(x_{i} \mid \omega\right) \approx H_{2}\left(x_{i} \mid 2 \omega_{o}\right)$ was used as the first-order approximation of the vibration FRF of the transformer.

Based on this approximation, previous work on mechanically and electrically excited FRFs of a model power transformer was extended to the study of FRF variations due to transformer mechanical faults. ${ }^{13}$ Research focusing on this work featured a sensitivity analysis of the mechanically and electrically excited FRFs of a model transformer to the causes of faults. Experimental evidence was presented to show the quantitative description of the causes of artificial faults and to extract features of variations of FRFs that might be useful to the vibration-based detection of the causes of transformer faults in general.

\section{THEORETICAL BACKGROUND}

The vibration response at location $x_{i}$ with respect to a distributed force excitation in the transformer mechanical system can be expressed as:

$$
v_{0}\left(x_{i} \mid \omega\right)=\int_{V} H_{M}\left(x_{i}, x_{k} \mid \omega, \xi_{M}\right) \hat{F}\left(x_{k} \mid \omega\right) d x_{k}
$$

where $H_{M}\left(x_{i}, x_{k} \mid \omega, \xi_{M}\right)$ was the mechanical FRF between $x_{i}$ and $x_{k}$, and $\hat{F}\left(x_{k} \mid \omega\right)$ was the force per unit volume at location $x_{k}$, and $V$ was the entire volume of the transformer structure. Unlike the traditional definition of an FRF, a mechanical parameter vector $\xi_{M}=\left[\xi_{M 1}, \xi_{M 2}, \ldots, \xi_{M P}\right]$ was used to describe the causes of a transformer's faults. Following the same logic, the distributed force could also be described by an electrical FRF that relates $\hat{F}\left(x_{k} \mid \omega\right)$ as an output and the sinusoidal voltage $U\left(\omega_{o}\right)$ as an input. As a result of this analysis, the firstorder approximation of the vibration FRF of the transformer (as described in Eq. (1)) was expressed as:

$H\left(x_{i} \mid \omega, \xi_{M}, \xi_{E}\right)=\int_{V} H_{M}\left(x_{i}, x_{k} \mid \omega, \xi_{M}\right) H_{E}\left(x_{k} \mid \omega, \xi_{E}\right) d x_{k} ;$

where $\xi_{E}=\left[\xi_{E 1}, \xi_{E 2}, \ldots, \xi_{E Q}\right]$ was an electrical parameter vector. How to relate the parameters in the mechanical and electrical parameter vectors to the causes of transformer failures and what was the sensitivity of $H$ with respect to the changes in $\xi_{M}$ and $\xi_{E}$ were the challenging questions for vibration-based fault diagnosis of power transformers. The variation of $H$, with respect to the system parameters, could be expressed as:

$$
\delta H\left(x_{i} \mid \omega, \xi_{M}, \xi_{E}\right)=\sum_{p=1}^{P} \frac{\partial H}{\partial \xi_{M p}} \delta \xi_{M p}+\sum_{q=1}^{Q} \frac{\partial H}{\partial \xi_{E q}} \delta \xi_{E q} .
$$

For the electrically excited FRF, which was mostly relevant to the input/output relationship of an in-service transformer, the variation of the mechanically excited FRF of the model power transformer with only the mechanical parameters could be evaluated. It should be noted here that the vibration-based method for detecting the causes of faults was mainly focused on those causes related to the mechanical parameters. Although changes in the electrical parameters would also cause variations in the electrical FRF of the transformer, such variations often occurred at a much higher frequency range and other techniques, such as FRA, were developed for such detection. If the transformer was excited by a point force at $x_{o}$, then this mechanically excited FRF could be expressed by using a volume integration of $H_{M}\left(x_{i}, x_{k} \mid \omega, \xi_{M}\right)$, as shown in Eq. (2). Its variation with the mechanical parameter vector was:

$$
\delta H_{M}\left(x_{i}, x_{o} \mid \omega, \xi_{M}\right)=\sum_{p=1}^{P} \frac{\partial H_{M}}{\partial \xi_{M p}} \delta \xi_{M p}
$$

The relationship between the variation of the mechanically excited FRF in Eq. (5) and that of the electrically excited FRF in Eq. (4) were found by expanding the first term on the righthand side of Eq. (4):

$\sum_{p=1}^{P} \frac{\partial H}{\partial \xi_{M p}} \delta \xi_{M p}=\int_{V}\left[\sum_{p=1}^{P} \frac{\partial H_{M}}{\partial \xi_{M p}} \delta \xi_{M p}\right] H_{E}\left(x_{k} \mid \omega, \xi_{E}\right) d x_{k}$

It was indicated that the variation of $\delta H\left(x_{i} \mid \omega, \xi_{M}, \xi_{E}\right)$ with respect to $\xi_{M}$ was the spatially averaged sensitivity of $H_{M}\left(x_{i}, x_{k} \mid \omega, \xi_{M}\right)$ over all of the forcing locations and weighted by $H_{E}\left(x_{k} \mid \omega, \xi_{E}\right)$.

\section{DESCRIPTION OF EXPERIMENTS}

The measurement of vibration responses was performed on a 10-kVA single-phase transformer with rating voltages of $415 / 240 \mathrm{~V}$. The experimental set-ups were kept the same as in a previous article (Wang and Pan), where the mechanical and electrical excitations were implemented by an impact force and swept-sine voltage, respectively. ${ }^{13}$ A sinusoidal voltage signal from a signal generator (Agilent, 33120A) was amplified by using a power amplifier (Yamaha, P2500S), and then a variac. As a result, a 200-V voltage at each test frequency was applied to the primary input of the model transformer. The transformer vibrations at 48 measurement locations were measured by accelerometers (IMI, 320A). The outputs of the accelerometers were pre-amplified by a signal conditioning device before being sent to a laptop computer for post-processing by using a DAQ (NI, USB-6259). In order to stay consistent with the previous study, the same impact location and test points were used.

To describe the causes of mechanical failures of a model power transformer using the mechanical parameter vector $\xi_{M}$, the percentage looseness of winding clamping pressure, and the percentage looseness of core clamping pressure are used as two independent components in $\xi_{M}$. For example, to describe the changes in the winding clamping force, the first element in $\xi_{M}$ is defined as $\xi_{M 1}=\xi_{M 1}^{(0)}\left(1-\eta_{1}\right)$, which changes the nominal value of $\xi_{M 1}^{(0)}$ (set by the manufacturer) to zero, where $0 \leq \eta_{1} \leq 1$ was the percentage looseness of the 


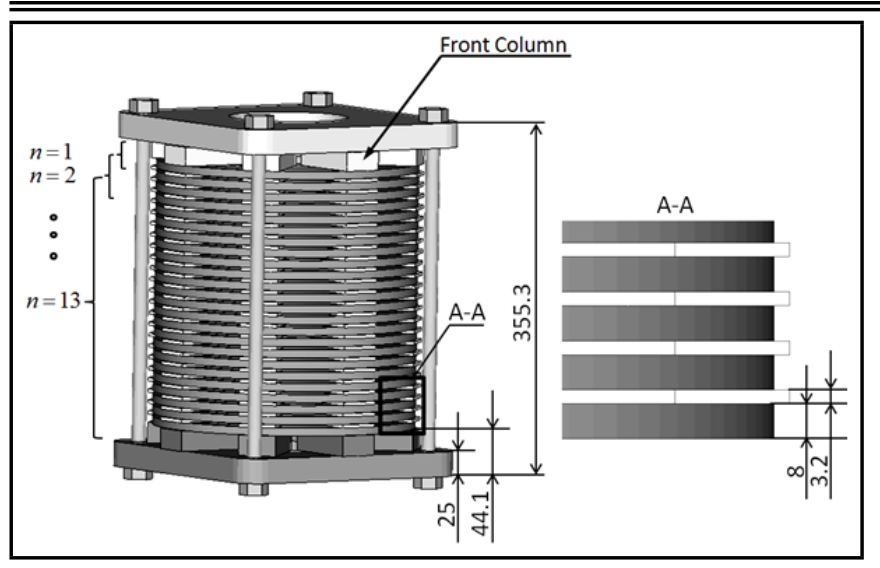

Figure 1. The design of longitudinal insulation and the arrangement of missing insulation spacers as a cause of mechanical faults.

core. Similarly, the winding clamping force was described by $\xi_{M 2}=\xi_{M 2}^{(0)}\left(1-\eta_{2}\right)$ with $0 \leq \eta_{2} \leq 1$.

For this model transformer, its winding and core segments were fastened by four and eight bolts respectively. ${ }^{13}$ The nominal clamping forces set by Universal Transformer were approximately $2250 \mathrm{~N}$ in the winding and $1500 \mathrm{~N}$ in the core. These two values were converted from the tightening torque by considering the bolt diameters, thread lead angle, etc. ${ }^{14}$ Realizing the aforementioned mechanical failures in terms of clamping looseness depends upon the gradual adjustment of the preloading of the corresponding clamping bolts using a torque wrench. Considering the importance of diagnosis a fault in its early stages, a maximum $35 \%$ clamping looseness in the winding and $25 \%$ looseness in the core were investigated with $\delta \eta=5 \%$ increments in both winding and core looseness.

In addition to the clamping looseness, the absence of the longitudinal insulation spacers was examined as another cause of mechanical faults, as these would reduce the axial stability and cause excessive vibration. The design of longitudinal insulation and arrangement of its mechanical faults, $\xi_{M 3}$, are presented in Fig. 1, where eight columns of insulation spacers are circumferentially equispaced along the winding circle.

The detailed dimensions of insulation spacers and winding conductors can be found in the partial view (A-A), where the height of the winding conductor and insulation spacer were $8 \mathrm{~mm}$ and $3.2 \mathrm{~mm}$, respectively. In addition to the thin dovetail spacers between the winding disks, an array of $19.1 \mathrm{~mm}$ thick insulation blocks were layered at both ends of the winding assembly. The fault of missing insulation spacers was introduced in the front column, including the insulation blocks at both ends, as indicated in Fig. 1. This column of insulation spacers was separated into thirteen segments with approximately equal height, corresponding to the thirteen missing insulation statuses of $\xi_{M 3}^{(n)}$ shown in Fig. 1. The insulation spacers were removed cumulatively from $n=1$ to $n=13$. All the missing-insulation tests were conducted under the same winding clamping status.

\section{RESULTS AND DISCUSSION}

Generally speaking, the development of mechanical faults in a transformer structure is accompanied by changes in the structural stiffness, mass, and damping. The resulting variations in a transformer's vibration responses due to some common faults will be presented in the following subsections.

\subsection{Vibration Changes Due to Core Looseness}

The first cause of mechanical failure under investigation was looseness in the transformer core clamping force, which was described by $\xi_{M 1}=\xi_{M 1}^{(0)}\left(1-\eta_{1}\right)$ with $0 \leq \eta_{1} \leq 1$. By gradually reducing the clamping force with the same percentage increment $\left(\delta \eta_{1}=5 \%\right)$, an overall $25 \%$ looseness was introduced to the left and right limbs symmetrically. Based on the experimental methodology described in a previous article, the vibration FRFs under mechanical and electrical excitations were measured. ${ }^{13}$ Spatially averaged FRFs with different core clamping forces are presented in Fig. 2, where a $5 \mathrm{~dB}$ offset from the FRF underneath is introduced for clarity. On each FRF curve, four resonance peaks can be clearly discerned under electrical and mechanical excitations. However, the resonance peaks at the $3^{\text {rd }}$ and $4^{\text {th }}$ mode responses were greatly affected due to core looseness, while the other modes seemed unaffected.

A quantitative comparison of the changes in vibration at different clamping status, by means of natural frequency shifts $\left(\delta f_{n}\right)$ and cumulative changes in the FRFs at $100 \mathrm{~Hz}$ $\left(\left.\delta H_{M}\right|_{\omega=100 \mathrm{~Hz}}\right.$ and $\left.\left.\delta H\right|_{\omega=100 \mathrm{~Hz}}\right)$, can be found in Table 1. The percentage of natural frequency shifts were calculated around the lowest state $\left(\eta_{1}=0\right)$ without core clamping looseness so as to give the cumulative changes of the frequency responses at $100 \mathrm{~Hz}$. Variations in vibration at discrete frequencies, i.e., $100 \mathrm{~Hz}$ and its harmonics, were of most concern in response-based monitoring strategies. Since the fundamental frequency at $100 \mathrm{~Hz}$ was very close to the $4^{\text {th }}$ core-controlled mode, the variation of the vibration component at $100 \mathrm{~Hz}$ was selected for a detailed analysis.

From Table 1, a general decrease of the first four natural frequencies is observed in the presence of the growing clamping looseness from $\eta_{1}=5 \%$ to $\eta_{1}=25 \%$. However, the frequency responses at $100 \mathrm{~Hz}$ increased dramatically under both excitations. To facilitate the explanation of these variations, the mode shapes of each resonance frequency are recalled in Fig. 2(a) based on the previous reports. ${ }^{13}$ According to the modal participation at each mode, the $1^{\text {st }}, 3^{\text {rd }}$, and $4^{\text {th }}$ modes could be classified as core-controlled modes, which are dominated by the transformer core assembly. The occurrence of clamping looseness directly affects the core-controlled modes owing to the resulting stiffness reduction in the core assembly. With the development of core looseness, the natural frequencies of the core-controlled modes were consistently reduced within the tested looseness range. As seen in Table 1, a maximum $10.19 \%(9.5 \mathrm{~Hz})$ frequency shift was recorded at the $4^{\text {th }}$ mode, which was really important for vibration-based condition monitoring. By using the modal parameter identification approach, the natural frequency shift could be detected and related to possible structural damages.

In contrast, the $2^{\text {nd }}$ mode appeared to be unaffected by the core looseness. The underlying reason for this was the modal participation, where the $2^{\text {nd }}$ mode was dominated by the trans- 


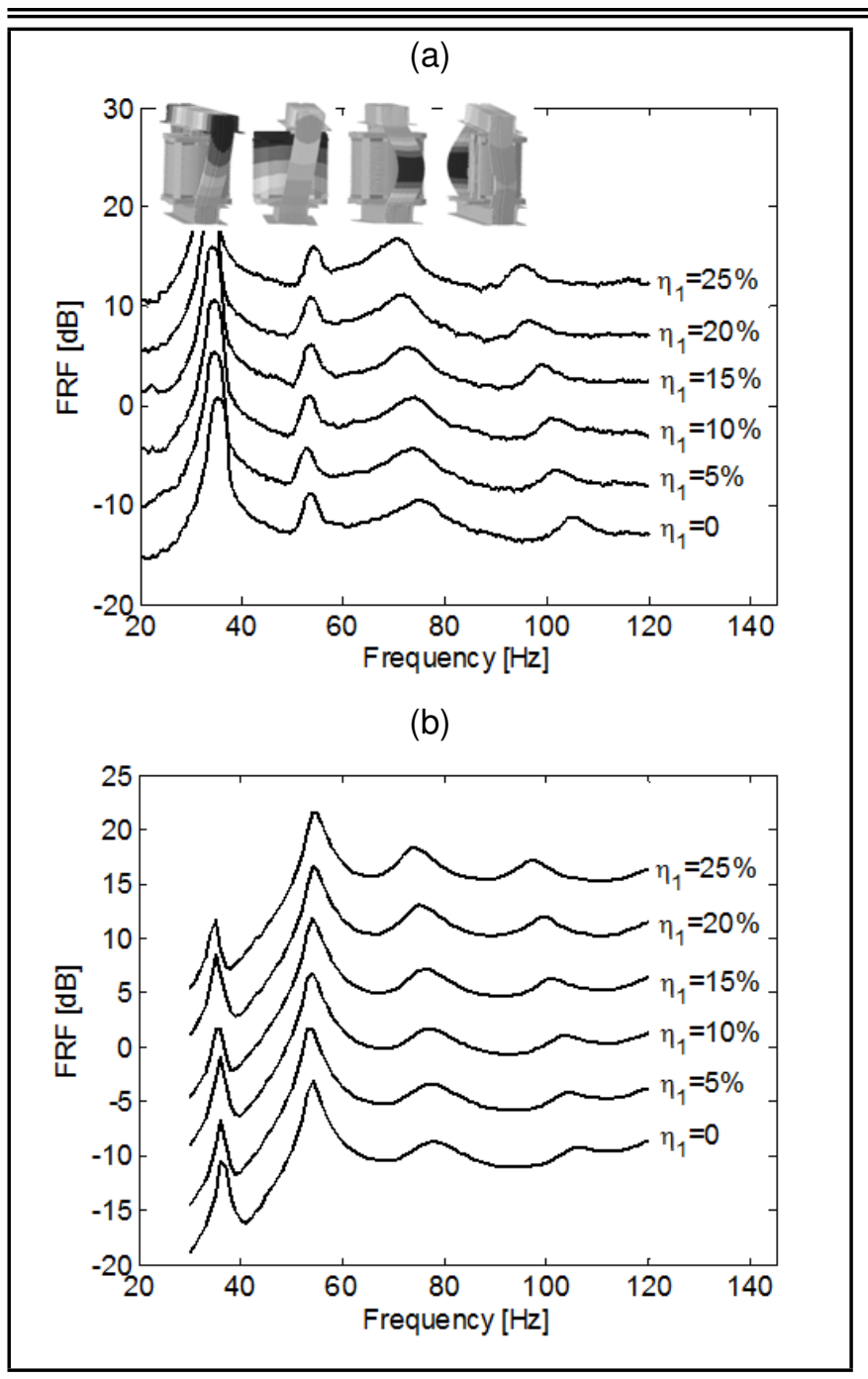

Figure 2. Spatially averaged FRFs of the transformer vibration due to (a) mechanical and (b) electrical excitations with core clamping looseness.

former winding. Since the transformer core also participated in this mode, the $2^{\text {nd }}$ mode was classified as a coupled mode between the core and winding assemblies. The orthogonality of vibration modes determines what causes of failure in the transformer core will not have much effect on the windingdominated mode, i.e., the $2^{\text {nd }}$ mode in this study.

The above experimental results verify that core looseness is able to change more than one mode in the low-frequency range. The dependency of natural frequency shifts on the causes of structural damage could potentially be employed for damage location. In addition to the frequency shift analyzed above, the deviations of both mechanically and electrically excited FRFs at $100 \mathrm{~Hz}$ due to core clamping looseness are listed in Table 1, where a maximum $2.56 \mathrm{~dB}$ increased under electrical excitation is observed. As another "side-effect" of stiffness reduction, an increase in the amplitude of the FRF was expected. Attention should be paid to the frequency shifts, which would increase the vibration response when a resonance peak was approaching and vice versa.

However, the variation under two excitations exhibits different sensitivities. The deviation of the electrically excited FRFs $(\delta H)$ depends not only on the stiffness changes but also on the magneto-mechanical coupling during magnetization of the transformer core. With clamping looseness, the internal stress
Table 1. Quantitative variation of the transformer vibration FRFs due to core clamping looseness.

\begin{tabular}{||c|c|c|c|c|c|c||}
\hline \multicolumn{2}{|c|}{$\eta_{1}(\%)$} & 5 & 10 & 15 & 20 & 25 \\
\hline \multirow{3}{*}{$\delta f_{n}(\%)$} & $1^{\text {st }}$ mode & -1.43 & -2.85 & -2.85 & -4.28 & -5.71 \\
\cline { 2 - 7 } & $2^{\text {nd }}$ mode & -0.94 & -0.94 & -0.94 & -0.94 & 0 \\
\cline { 2 - 7 } & $3^{\text {rd }}$ mode & -1.3 & -2.6 & -3.25 & -4.55 & -5.19 \\
\cline { 2 - 7 } & $4^{\text {th }}$ mode & -3.4 & -4.37 & -6.8 & -8.74 & -10.19 \\
\hline$\delta H_{M}(\mathrm{~dB})$ & $\omega=100 \mathrm{~Hz}$ & 1.15 & 1.49 & 2.04 & 0.74 & 0.69 \\
\hline$\delta H(\mathrm{~dB})$ & $\omega=100 \mathrm{~Hz}$ & 0.51 & 0.91 & 1.77 & 2.56 & 2.12 \\
\hline
\end{tabular}

Table 2. Quantitative variation of the vibration FRFs due to winding clamping looseness.

\begin{tabular}{|c|c|c|c|c|c|c|c|c|}
\hline \multicolumn{2}{|c|}{$\eta_{2}(\%)$} & 5 & 10 & 15 & 20 & 25 & 30 & 35 \\
\hline \multirow{4}{*}{$\delta f_{n}(\%)$} & $1^{\text {st }}$ mode & -1.4 & -1.4 & -1.4 & -1.4 & -1.4 & -1.4 & -1.4 \\
\cline { 2 - 9 } & $2^{\text {nd }}$ mode & -1.9 & -3.8 & -5.7 & -6.6 & -9.4 & -10.4 & -11.3 \\
\cline { 2 - 9 } & $3^{\text {rd }}$ mode & -0.6 & -0.6 & -0.6 & 0 & 0 & 0 & -0.6 \\
\cline { 2 - 9 } & $4^{\text {th }}$ mode & -1.5 & -0.9 & -0.5 & -0.5 & -0.9 & -1.94 & -1.94 \\
\hline$\delta H_{M}(\mathrm{~dB})$ & $50 \mathrm{~Hz}$ & 0.44 & 2.43 & 3.65 & 3.7 & 2.39 & 1.97 & 2.09 \\
\hline$\delta H(\mathrm{~dB})$ & $50 \mathrm{~Hz}$ & 1.53 & 2.19 & 4.07 & 4.52 & 4.75 & 4.24 & 3.62 \\
\hline
\end{tabular}

of the silicon steel laminations is reduced as well, which leads to a weaker magneto-mechanical coupling and thus results in a smaller magnetostriction. ${ }^{15,16}$ This is another factor influencing the electrically excited FRFs, as core clamping looseness will decrease the magnetostriction. Combined with the above factors, the synthetic effect is to increase the electrically excited FRFs due to core looseness in this study. It is also worth mentioning that the vibration response under electrical excitation is more sensitive to core looseness, as can be concluded from Table 1 .

\subsection{Vibration Changes Due to Winding Looseness}

Transformer winding looseness is the second cause of mechanical failures in the model transformer to be studied. Winding looseness is described by $\xi_{M 2}=\xi_{M 2}^{(0)}\left(1-\eta_{2}\right)$ with $0 \leq \eta_{2} \leq 1$ to represent different clamping statuses. By gradually reducing the clamping force with the same percentage increment $\left(\delta \eta_{2}=5 \%\right)$, an overall $35 \%$ looseness was introduced to the transformer winding. The mechanically and electrically excited FRFs were measured using the same test equipment in Section 4.1. Figure 3 shows the spatially averaged FRFs under different winding clamping forces. A $5 \mathrm{~dB}$ offset from the FRF underneath is also introduced to clarify the picture.

As can be seen in Fig. 3, four natural frequencies can be recognized from the electrical and mechanical FRFs below $120 \mathrm{~Hz}$. The difference comes from their variations in the presence of winding clamping looseness. When the mechanical parameter vector changed from $\eta_{2}=0$ to $\eta_{2}=35 \%$ by reducing the winding clamping forces, a gradual decrease occured at the $2^{\text {nd }}$ natural frequency. Meanwhile, the natural frequencies of the other modes appeared to be unaffected. Compared with the variation of the FRFs in the presence of core looseness, this phenomenon was regarded as one of the characteristics for winding anomalies. Similarly, the quantitative variation of the vibration FRFs due to winding clamping looseness is summarized in Table 2. Since the most affected mode was the $2^{\text {nd }}$ mode at $53 \mathrm{~Hz}$, the frequency response at $50 \mathrm{~Hz}$ was analyzed as well as the natural frequency shift.

As can be seen in Table 2, the appearance of winding clamp- 


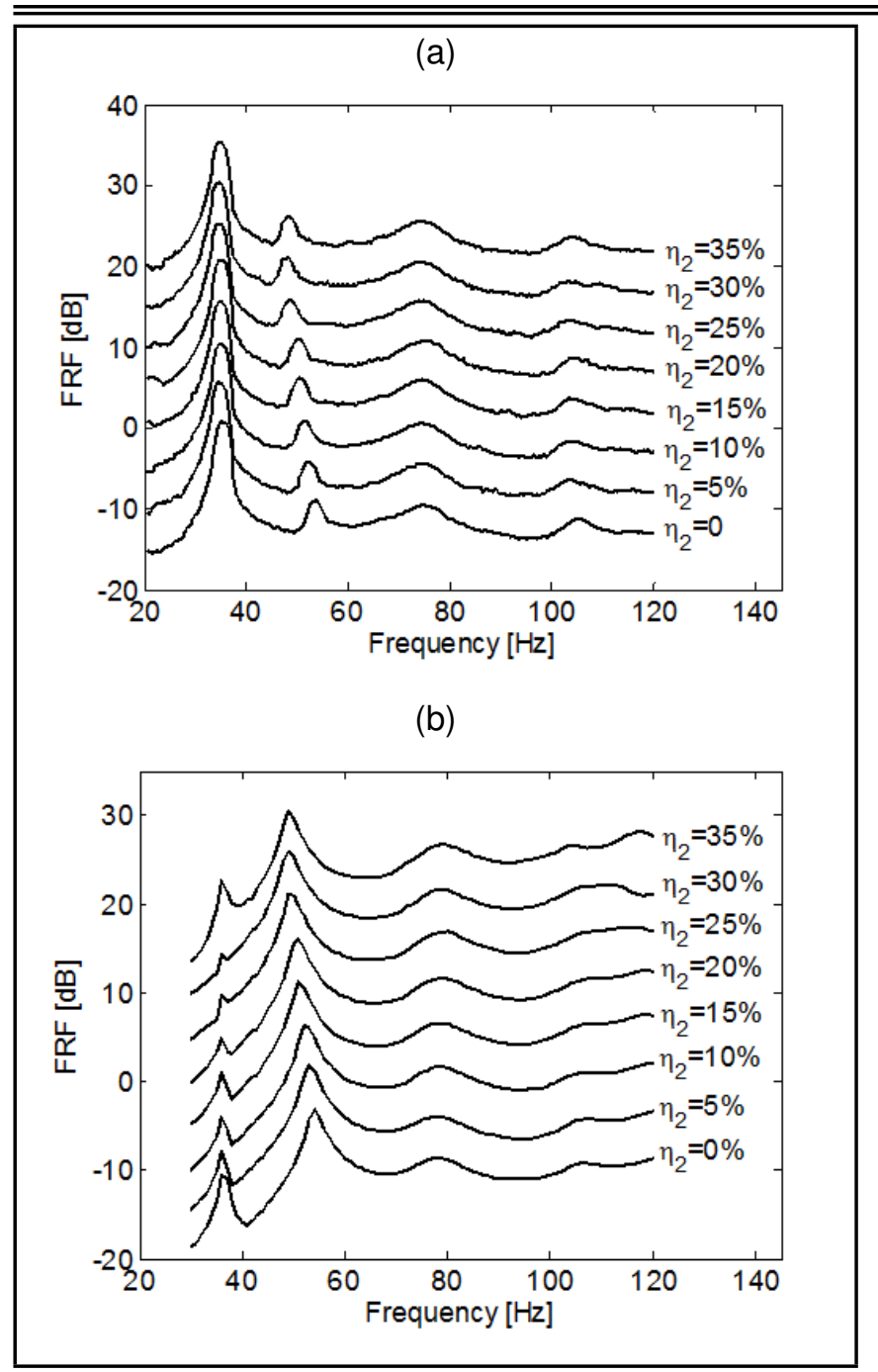

Figure 3. Spatially averaged FRF of the transformer vibration due to (a) mechanical and (b) electrical excitations with winding clamping looseness.

ing looseness leads to a general decrease of all four natural frequencies. The most affected mode was the $2^{\text {nd }}$ mode at $53 \mathrm{~Hz}$, which caused about a $11.32 \%(6 \mathrm{~Hz})$ decrease at maximum looseness. This experimental result was fully expected since the aforementioned causes of the failures were a natural reduction in the transformer's local stiffness. As to why the frequency shift occured at the $2^{\text {nd }}$ mode, the answer was found from the modal analysis, where the $2^{\text {nd }}$ mode was dominated by the winding assembly. The winding clamping looseness mainly caused a stiffness reduction in the winding rather than the core assembly. Since the natural frequencies were measured in exactly the same way as in the mechanical and electrical excitation cases, the same trend in frequency shift was observed in the electrically excited cases.

Apart from the analysis of natural frequency shifts, amplitude variations of the FRFs due to winding looseness were also examined in both the mechanically and electrically excited cases. The deviations calculated in Table 2 were also cumulative changes relative to the lowest state without looseness $\left(\eta_{2}=0\right)$. Compared to the vibration FRF of the initial clamping state, a general increase at $50 \mathrm{~Hz}$ was found in both the mechanically and electrically excited cases in the presence of winding looseness. In particular, the vibration response at $50 \mathrm{~Hz}$ first increased with the approach of the $2^{\text {nd }}$ natural fre- quency and then decreased as it moved further away. The overall increase in the vibration response could be understood as a result of the reduction of the stiffness in the winding assembly.

Since the vibration test was performed in a transformer under no-load conditions, the electromagnetic (EM) force in the winding was caused by the interaction between the magnetizing current and the leakage field. The magnetizing current was only a few hundred milliamps and the resultant EM force was relatively small. Excitation caused by EM forces in the winding was very weak and could be neglected compared to core excitation. Therefore, the excitation force in these cases remained almost the same, and was mainly composed of magnetostrictive force in the core. In other words, the changes in mechanical properties induced by a structural anomaly were responsible for the variations of the vibration FRFs. Maximum increases of $3.7 \mathrm{~dB}$ and $4.75 \mathrm{~dB}$ were measured at $50 \mathrm{~Hz}$ in the mechanical and electrical FRFs, respectively. Such obvious deviations were more than enough to be detected in the vibration response-based monitoring methods.

\subsection{Vibration Changes Due to Missing Insulation Spacers}

The third case study is dedicated to one of the causes of insulation faults in the winding assembly. As described in Fig. 1, insulation damage was simulated by removing a small portion of the insulation spacers along the longitudinal direction. In this study, the absence of insulation spacers was catalogued as a mechanical property change, since it indeed altered the mechanical integrity of the transformer structure. The spatially averaged FRFs of the transformer vibration with missing insulation spacers in the winding insulation system are presented in Fig. 4.

No obvious frequency shifts in this frequency range could be found by visually examining the mechanically and electrically excited FRFs, even when all the front insulation spacers were removed ( $n=13$ ). To quantitatively analyse the variation of the FRFs due to missing insulation spacers, a detailed summary of these frequency shifts is listed in Table 3. Since the missing insulation spacers were introduced in the winding assembly and the $2^{\text {nd }}$ mode at $53 \mathrm{~Hz}$ was dominated by this component, the vibration response at $50 \mathrm{~Hz}$ is also presented in Table 3. The maximum frequency shift in this case was $2.5 \mathrm{~Hz}$ $(4.71 \%)$ at the $2^{\text {nd }}$ mode, while the other modes appeared unaffected. The underlying reason was also attributed to stiffness reduction in the winding.

The analysis of extracted data verified that although the frequency shifts were small, the amplitude increases at $50 \mathrm{~Hz}$ were pronounced; they were $2.29 \mathrm{~dB}$ and $3.47 \mathrm{~dB}$ in the mechanical and electrical FRFs, respectively. These results imply that the amplitude of the frequency response at certain frequencies could be altered dramatically, even with a small frequency shift when there was a resonance frequency nearby. Similar to the winding looseness case, these variations in the FRFs were merely caused by changes in the mechanical property rather than by excitation differences.

It was found that a structural anomaly in the core could produce considerable variations in the low-frequency range. Al- 


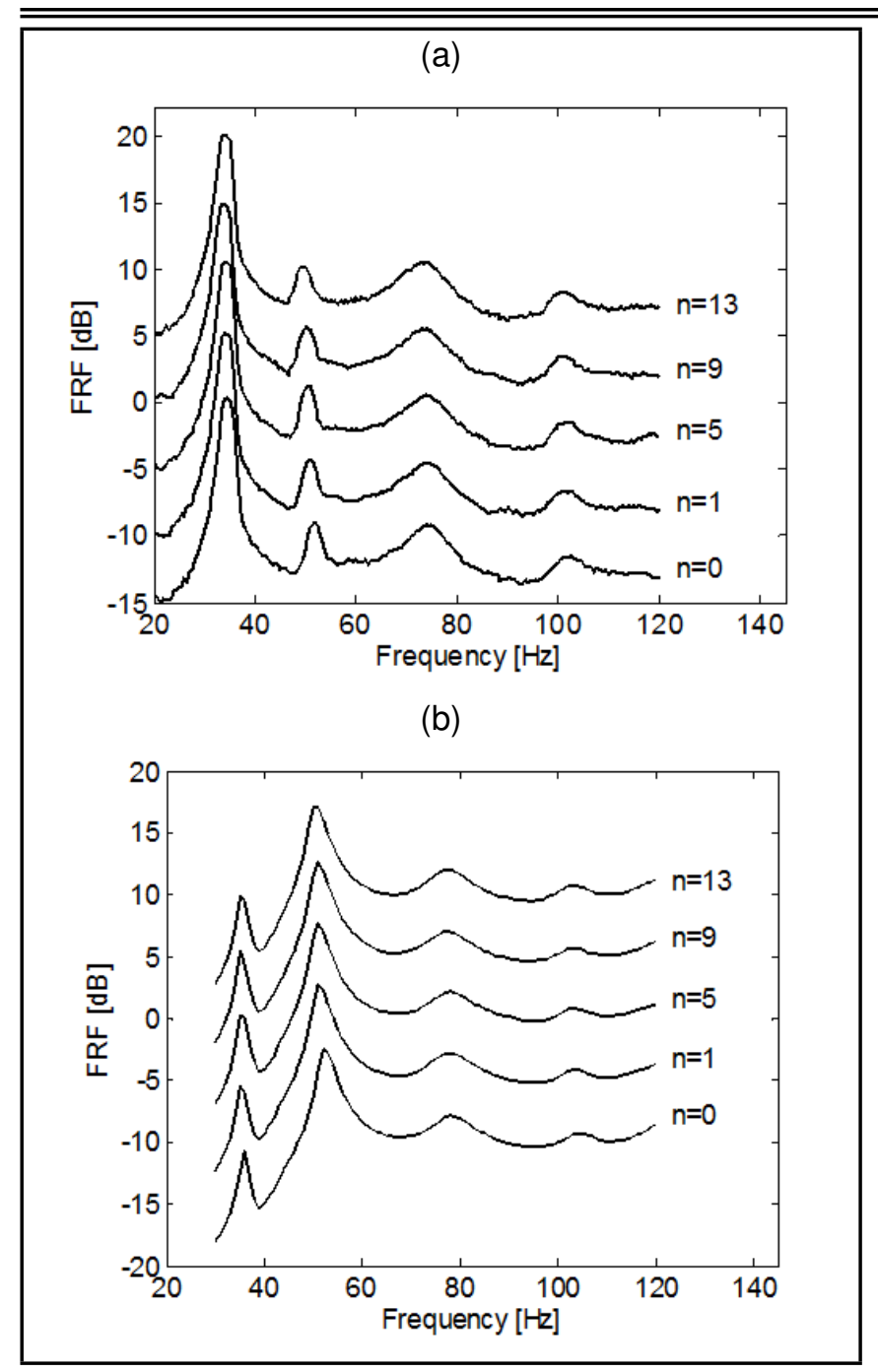

Figure 4. Spatially averaged FRFs of the transformer vibration due to (a) mechanical and (b) electrical excitations with missing insulation spacers.

though the amplitude of the FRFs changed dramatically as a result of winding anomalies, i.e., missing insulation spacers, the sensitivity of the natural frequency shift was not high. As can be seen in Fig. 2(a), although the coupled mode at $53 \mathrm{~Hz}$ was dominated by the transformer winding, it was actually the rigid-body movement around the core bottom yoke. The clamping looseness and missing insulation spacers affected the connection boundaries in this coupled mode and thus gave rise to the above variations in the FRFs. However, the most affected modal response was anticipated to be at the winding-controlled modes in the higher frequency range. According to the previous modal analysis on the same transformer, the windingcontrolled modes were in a frequency range of $>200 \mathrm{~Hz}{ }^{13}$ To verify this speculation, the variation of the FRFs between $120 \mathrm{~Hz}$ and $1000 \mathrm{~Hz}$ were investigated in the following section.

Table 3. Quantitative variation of the transformer vibration FRFs due to missing insulation spacers.

\begin{tabular}{||c|c|c|c|c|c|}
\hline \multicolumn{2}{|c|}{$n$} & 1 & 5 & 9 & 13 \\
\hline \multirow{3}{*}{$\delta f_{n}(\%)$} & $1^{\text {st }}$ mode & 0 & 0 & 0 & -1.43 \\
\cline { 2 - 6 } & $2^{\text {nd }}$ mode & -1.9 & -2.83 & -3.77 & -4.71 \\
\cline { 2 - 6 } & $3^{\text {rd }}$ mode & 0 & 0 & 0 & -0.65 \\
\cline { 2 - 6 } & $4^{\text {th }}$ mode & 0 & 0 & -0.49 & 0 \\
\hline$\delta H_{M}(\mathrm{~dB})$ & $f=50 \mathrm{~Hz}$ & 0.76 & 1.97 & 2.0 & 2.29 \\
\hline$\delta H(\mathrm{~dB})$ & $f=50 \mathrm{~Hz}$ & 2.51 & 2.99 & 3.04 & 3.47 \\
\hline
\end{tabular}

(a)

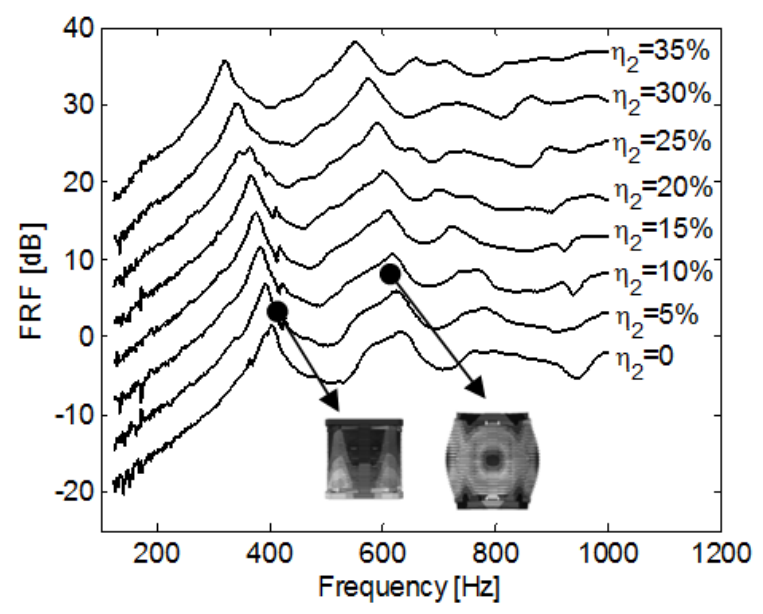

(b)

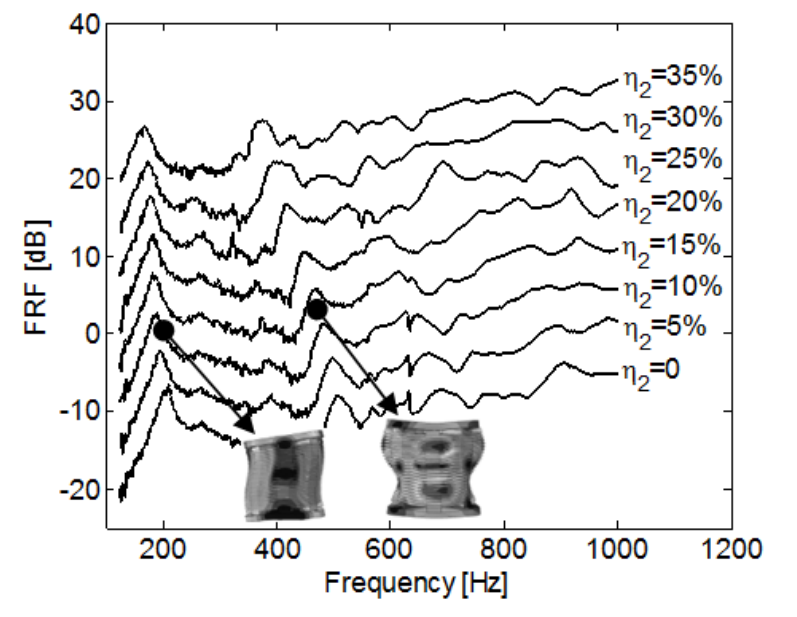

Figure 5. Spatially averaged FRFs of the transformer vibration due to winding looseness in the (a) radial and (b) axial directions.

\subsection{Variation of the High-frequency Vibration Response}

As was seen in the above discussion, the natural frequencies obtained under the electrical excitation were the same as under the mechanical excitation. To study the frequency shift due to winding anomalies, the FRFs of the mechanical excitation are selected for analysis. The electrical excitation case was not examined due to the lack of a high-voltage source with variable frequencies.

\subsubsection{Variation of high-frequency response to wind- ing looseness}

To study the vibration changes in a higher frequency range, the radial and axial vibration modes were first examined to classify whether they are winding-controlled modes. The spatially averaged FRFs due to the different winding clamping forces are presented in Fig. 5, where the mode shapes for specific resonances are shown as well. The winding-controlled modes at around $200 \mathrm{~Hz}, 400 \mathrm{~Hz}, 500 \mathrm{~Hz}$, and $600 \mathrm{~Hz}$ were all dominated by the winding assembly, where the participation of the core could be neglected. As can be seen from Fig. 5, the natural frequencies at these four modes all decreased with the development of winding clamping looseness. 
Table 4. Natural frequency shifts $\left(\delta f_{n}\right)$ of the winding-controlled modes due to looseness in the winding clamping force.

\begin{tabular}{|c|c|c|c|c|c|c|c|c|}
\hline \multicolumn{2}{|c|}{$\eta_{2}(\%)$} & 5 & 10 & 15 & 20 & 25 & 30 & 35 \\
\hline \multirow{4}{*}{$\begin{array}{c}\text { Radial } \\
\text { modes }\end{array}$} & $2^{\text {st }}(\mathrm{Hz})$ & -12 & -28 & -35 & -40 & -53 & -63 & -75 \\
\cline { 2 - 9 } & $2^{\text {nd }}(\mathrm{Hz})$ & -13 & -28 & -42 & -50 & -58 & -70 & -86 \\
\cline { 2 - 9 } & $1^{\text {st }}(\%)$ & -2.8 & -6.8 & -8.9 & 10.3 & -13.8 & -17 & -20.8 \\
\cline { 2 - 9 } & $2^{\text {nd }}(\%)$ & -1.9 & -4.3 & -6.6 & -8 & -9.4 & -11.6 & -14.5 \\
\hline \multirow{4}{*}{$\begin{array}{c}\text { Axial } \\
\text { modes }\end{array}$} & $2^{\text {st }}(\mathrm{Hz})$ & -12 & -21 & -26 & -29 & -31 & -33 & -35 \\
\cline { 2 - 9 } & $2^{\text {nd }}(\mathrm{Hz})$ & -11 & -26 & -41 & -59 & -91 & -114 & -137 \\
\cline { 2 - 9 } & $1^{\text {st }}(\%)$ & -5.1 & -9.4 & -12.2 & -13.9 & -15 & -16.1 & -17.3 \\
\cline { 2 - 9 } & $2^{\text {nd }}(\%)$ & -2.1 & -4.9 & -8.1 & -11.9 & -19.2 & -25.7 & -32.6 \\
\hline
\end{tabular}

Table 5. Natural frequency shifts $\left(\delta f_{n}\right)$ of the winding-controlled modes due to missing insulation spacers.

\begin{tabular}{||c|c|c|c|c|c|c|c|c|}
\hline \multicolumn{2}{|c|}{$n$} & 1 & 3 & 5 & 7 & 9 & 11 & 13 \\
\hline \multirow{4}{*}{$\begin{array}{c}\text { Radial } \\
\text { modes }\end{array}$} & $1^{\text {st }}(\mathrm{Hz})$ & -3 & -4 & -9 & -17 & -26 & -39 & -45 \\
\cline { 2 - 9 } & $2^{\text {nd }}(\mathrm{Hz})$ & -9 & -17 & -23 & -32 & -38 & -66 & -67 \\
\cline { 2 - 10 } & $1^{\text {st }}(\%)$ & -0.8 & -1.1 & -2.3 & -4.9 & -7.8 & -12.1 & -14.2 \\
\cline { 2 - 9 } & $2^{\text {nd }}(\%)$ & -1.5 & -2.9 & -4.0 & -5.7 & -6.8 & -12.3 & -12.8 \\
\hline \multirow{4}{*}{$\begin{array}{c}\text { Axial } \\
\text { modes }\end{array}$} & $1^{\text {st }}(\mathrm{Hz})$ & 0 & 1 & 0 & -3 & -4 & -8 & -8 \\
\cline { 2 - 9 } & $2^{\text {nd }}(\mathrm{Hz})$ & 2 & 3 & -3 & -16 & - & - & - \\
\cline { 2 - 9 } & $1^{\text {st }}(\%)$ & 0 & 0.5 & 0 & -0.17 & -0.23 & -4.6 & -4.6 \\
\cline { 2 - 9 } & $2^{\text {nd }}(\%)$ & 0.5 & 0.75 & -0.75 & -4.0 & - & - & - \\
\hline \hline
\end{tabular}

This observation was the same as in the low-frequency range. However, the sensitivity of the frequency shift to the winding looseness was much higher than that in the lowfrequency range. Table 4 specifies the corresponding frequency shifts of the relevant modes, where a maximum $32.6 \%$ $(137 \mathrm{~Hz})$ decrease in the natural frequency could be found for the $2^{\text {nd }}$ winding-controlled mode in the axial direction. The frequency shifts for the modes with large radial components were also remarkable, which reached a $20.8 \%$ reduction at $35 \%$ winding looseness.

\subsubsection{Variation of high-frequency response to miss- ing insulation spacers}

As reported in Section 4.3, the frequency shift due to missing insulation spacers was not obvious, since there was only one coupled mode in the analysed low-frequency range and it was not sensitive to the change in clamping force. Here, the investigation was extended to a higher frequency range from $120 \mathrm{~Hz}$ to $1000 \mathrm{~Hz}$, which covered four winding-controlled modes, as illustrated in Fig. 5. Given the high sensitivity of the frequency shift to looseness of the winding clamping force, finer test steps were adopted in this case study. Seven missing insulation statuses were equally spaced from $n=1$ to $n=13$ with the same amount of total missing spacers, as conducted in Section 4.3.

Figure 6 shows the spatially averaged FRFs between $120 \mathrm{~Hz}$ and $1000 \mathrm{~Hz}$ of transformer vibration for different amounts of missing insulation spacers. The shift of resonance peaks could be clearly discerned at the winding-controlled mode in the radial direction. Detailed percentage variations and absolute frequency shifts in hertz are summarized in Table 5. A maximum $14.2 \%(45 \mathrm{~Hz})$ decrease was measured at $n=13$ in the $1^{\text {st }}$ winding-controlled mode in the radial direction. However, the frequency shift did not appear to be obvious in the axial direction. For the axial mode at $400 \mathrm{~Hz}$, the frequency shifts at the last three statuses were not listed due to the local resonance after $n=7$. This indicated that reducing the clamping (a)

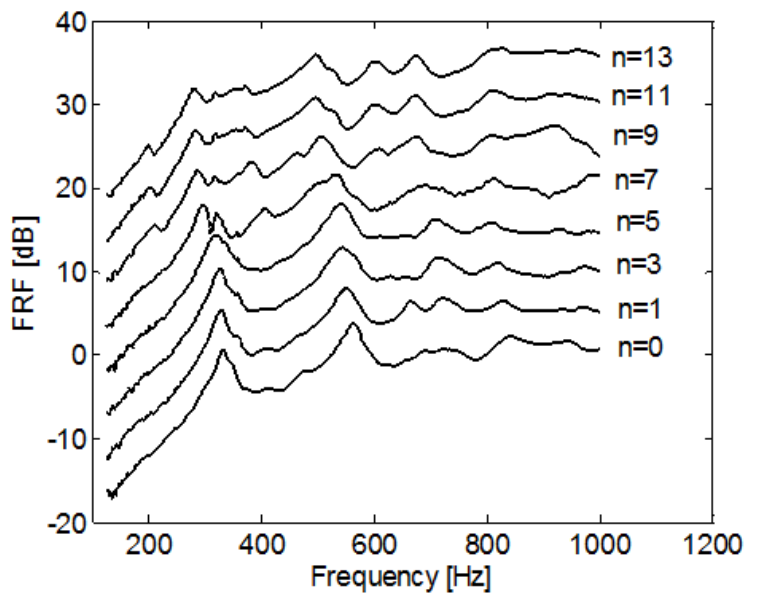

(b)

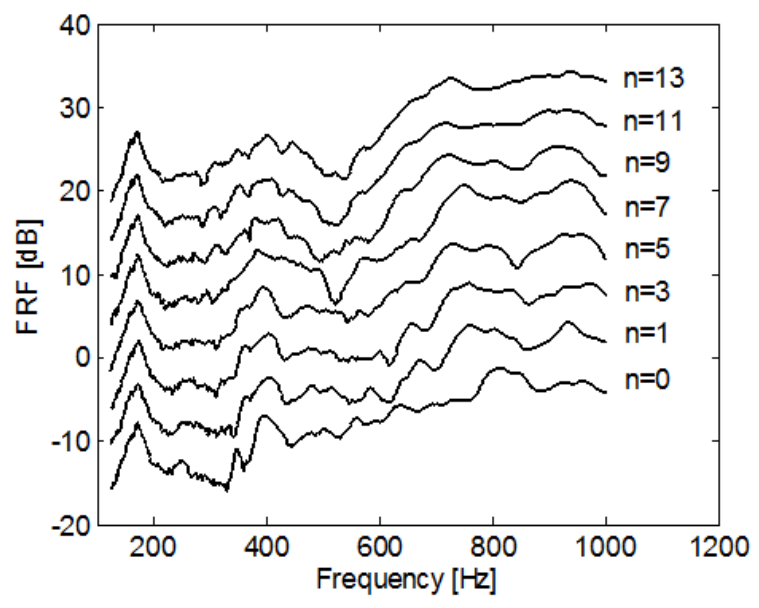

Figure 6. Spatially averaged FRFs of the transformer vibration due to missing insulation spacers in the (a) radial and (b) axial directions.

force would not only cause the shifts of natural frequencies, but also allow the observation of extra resonances in the FRF. This may be another interesting vibration feature that might be useful for transformer condition monitoring. Either frequency shifts or the appearance of extra resonance peaks could be related to the looseness of the clamping force.

\section{CONCLUSIONS}

In this paper, a single-phase 10-kVA model transformer was studied as an example to demonstrate the changes in its vibration response to several winding faults, namely looseness of clamping forces in the winding and in the core, and the absence of insulation spacers. The variations of the FRFs due to mechanical parameter changes were measured using the sweep-sine test and the impact test. For the three different causes of transformer faults, their influences on the vibration response were examined by comparing the structural frequency responses of the intact and "damaged" transformers. The underlying reasons for these variations were then analysed.

As expected, the faults were all capable of altering the mechanically and electrically excited FRFs. More specifically, the occurrence of winding and core looseness, as well as the ab- 
sence of insulation spacers decreased the relevant natural frequencies as a result of the loss of structural stiffness caused by the faults. The maximum $11.32 \%$ decrease in the lowfrequency range and $32.6 \%$ in the high-frequency range were measured in the presence of these causes of failure.

Compared to the low-frequency FRFs, higher sensitivities to variation were found in the high-frequency FRFs. Moreover, local resonances would be produced in the high-frequency range with the development of the causes of failure, as shown in the missing insulation spacer case.

\section{ACKNOWLEDGEMENTS}

The authors would like to thank the CRC for Infrastructure Engineering Asset Management (CIEAM) in Australia and State Grid Corporation of China for their financial support for this work.

\section{REFERENCES}

1 Lin, C. E., Ling, J. M. and Huang, C. L. An expert system for transformer fault-diagnosis using dissolved-gas analysis, IEEE Transactions on Power Delivery, 8 (1), 231-238, (1993). http://dx.doi.org/10.1109/61.180341

2 Leibfried, T. and Feser, K. Monitoring of power transformers using the transfer function method, IEEE Transactions on Power Delivery, 14 (4), 1333-1341, (1999). http://dx.doi.org/10.1109/61.796226

3 Bartoletti, C., Desiderio, M., Carlo, D. D., Fazio, G., Muzi, F., Sacerdoti, G., and Salvatori, F. Vibro-acoustic techniques to diagnose power transformers, IEEE Transactions on Power Delivery, 19 (1), 221-229, (2004). http://dx.doi.org/10.1109/TPWRD.2003.820177

4 Sun, H. C., Huang, Y. C., and Huang, C. M. A review of dissolved gas analysis in power transformers, Energy Procedia, 14, 1220-1225, (2012). http://dx.doi.org/10.1016/j.egypro.2011.12.1079

5 Gómez-Luna, E., Aponte, G., Gonzalez-Garcia, C., and Pleite, J. Current status and future trends in the frequency response analysis (FRA) with the transformer in service, IEEE Transactions on Power Delivery, 28 (2), 1024-1031, (2013). http://dx.doi.org/10.1109/TPWRD.2012.2234141

6 Berler, Z., Golubev, A., Rusov, V., Tsvetkov, V., and Patterson, C. Vibro-acoustic method of transformer clamping pressure monitoring, Conference Record of the 2000 IEEE International Symposium on Electrical Insulation, 263-266, (2000). http://dx.doi.org/10.1109/ELINSL.2000.845503
7 Mechefske, C. K. Correlating power transformer tank vibration characteristics to winding looseness, Insight, 37 (8), 599-604, (1995). http://dx.doi.org/10176.35400005402731.0030

8 Kornatowski, E. Mechanical-condition assessment of power transformer using vibroacoustic analysis, Key Engineering Materials, 500, 40-44, (2012). http://dx.doi.org/10.4028/www.scientific.net/KEM.500.40

9 Borucki, S. Diagnosis of technical condition of power transformers based on the analysis of vibroacoustic signals measured in transient operating conditions, IEEE Transactions on Power Delivery, 27 (2), 670-676, (2012). http://dx.doi.org/10.1109/TPWRD.2012.2185955

10 Wu, S., Huang, W., Kong, F., Wu, Q., Zhou, F., Zhang, R., and Wang, Z. Extracting power transformer vibration features by a time-scale-frequency analysis method, Journal of Electromagnetic Analysis and Applications, 2, 3138, (2010). http://dx.doi.org/10.4236/jemaa.2010.21005

11 Garcia, B., Burgos, J. C., and Alonso, A. M. Transformer tank vibration modeling as a method of detecting winding deformations-Part I: Theoretical foundation, IEEE Transactions on Power Delivery, 21 (1), 157-163, (2006). http://dx.doi.org/10.1109/TPWRD.2005.852280

12 Garcia, B., Burgos, J. C., and Alonso, A. M. Transformer tank vibration modeling as a method of detecting winding deformations-Part II: Experimental verification, IEEE Transactions on Power Delivery, 21 (1), 164-169, (2006). http://dx.doi.org/10.1109/TPWRD.2005.852275

13 Wang, Y. and Pan, J. Comparison of mechanically and electrically excited vibration frequency responses of a model power transformer, IEEE Transactions on Power Delivery, (2015). http://dx.doi.org/10.1109/TPWRD.2014.2383390

14 Zhang, Z., Ying, B., and Jiao, M. Discussions on the determination of bolt clamping forces, Chemical Engineering and Equipment, 8, 105-109, (2009). http://dx.doi.org/10.3969/j.issn.1003-0735.2009.08.035

15 Moses, A. J. Effects of stresses on magnetic properties of silicon-iron laminations, Journal of Material Science, 9 (2), 217-222, (1974). http://dx.doi.org/10.1007/BF00550944

16 Ilo, A., Weiser, B., Booth, T., and Pfützner, H. Influence of geometric parameters on the magnetic properties of model transformer cores, Journal of Magnetism and Magnetic Materials, 160, 38-40, (1996) 38-40. http://dx.doi.org/10.1016/0304-8853(96)00099-6 